\title{
World-Universe Model Predictions
}

\author{
Vladimir S. Netchitailo \\ Biolase Inc., 4 Cromwell, Irvine CA, USA \\ Email: netchitailov@gmail.com
}

How to cite this paper: Netchitailo, V.S. (2020) World-Universe Model Predictions. Journal of High Energy Physics, Gravitation and Cosmology, 6, 282-297. https://doi.org/10.4236/jhepgc.2020.62022

Received: March 2, 2020

Accepted: April 27, 2020

Published: April 30, 2020

Copyright $\odot 2020$ by author(s) and Scientific Research Publishing Inc. This work is licensed under the Creative Commons Attribution International License (CC BY 4.0).

http://creativecommons.org/licenses/by/4.0/ (c) (i) Open Access

\begin{abstract}
In 2013, World-Universe Model (WUM) proposed a principally different way to solve the problem of Newtonian Constant of Gravitation measurement precision. WUM revealed a self-consistent set of time-varying values of Primary Cosmological parameters of the World: Gravitation parameter, Hubble's parameter, Age of the World, Temperature of the Microwave Background Radiation, and the concentration of Intergalactic plasma. Based on the inter-connectivity of these parameters, WUM solved the Missing Baryon problem and predicted the values of the following Cosmological parameters: gravitation $G$, concentration of Intergalactic plasma, relative energy density of protons in the Medium, and the minimum energy of photons, which were experimentally confirmed in 2015-2018. Between 2013 and 2018, the relative standard uncertainty of $G$ measurements decreased x6. The set of values obtained by WUM was recommended for consideration in CODATA Recommended Values of the Fundamental Physical Constants 2014.
\end{abstract}

\section{Keywords}

World-Universe Model, Dimensionless Time-Varying Parameter $Q$, Gravitational Parameter, Hubble's Parameter, Age of the World, Temperature of Microwave Background Radiation, Temperature of Far-Infrared Background Radiation Peak, Medium of the World, Inter-Connectivity of Primary Cosmological Parameters, Multicomponent Dark Matter, Weak Interaction, Intergalactic Plasma, Neutrinos, CODATA

\section{Introduction}

It doesn't make any difference how beautiful your guess is, it doesn't make any difference how smart you are, who made the guess, or what his name is. If it disagrees with experiment, it's wrong. That's all there is to it.

Richard Feynman 
The very first "World-Universe Model" paper was published on viXra on March 2013. At that time, great results were achieved:

- The cosmic Far-Infrared Background (FIRB) was announced in 1999. FIRB is part of the Cosmic Infrared Background with wavelengths near 100 microns that is the peak power wavelength of black-body radiation at temperature $T_{\text {FIRB }}=29 \mathrm{~K} \quad[1]$;

- Microwave Background Radiation (MBR) temperature $T_{M B R}=2.72548 \pm 0.00057 \mathrm{~K}$ was measured in 2009 [2];

- Nine-Year Wilkinson Microwave Anisotropy Probe (WMAP) Observations were published in 2013. The WMAP mission has resulted in a highly constrained $\Lambda \mathrm{CDM}$ cosmological model with precise and accurate parameters in agreement with a host of other cosmological measurements [3] (see Section 2.1). In 2013, the most important for the Cosmology, Newtonian constant of gravitation $G$, proved too difficult to measure [4] (see analysis in Section 2.2). Its measurement precision was the worst among all Fundamental physical constants. In 2013 WUM proposed principally different way to solve the problem of $G$ measurement precision and made some predictions of values of Primary Cosmological parameters [5] [6] (see Section 3.1).

\section{Status of Primary Cosmological Parameters in 2013}

\subsection{WMAP Mission Results}

The Big Bang Model (BBM) offers a comprehensive explanation for a broad range of observed phenomena. The framework for the BBM relies on General Relativity and on simplifying assumptions such as homogeneity and isotropy of space. The Lambda Cold Dark Matter $(\Lambda \mathrm{CDM})$ model is a parametrization of the BBM in which the universe contains three major components: first, a Cosmological constant $\Lambda$ associated with Dark Energy; second, the postulated Cold Dark Matter (CDM); and third, Ordinary Matter.

The $\Lambda$ CDM model is based on six parameters: baryon density $\Omega_{B}$, dark matter density $\Omega_{D M}$, dark energy density $\Omega_{\Lambda}$, scalar spectral index, curvature fluctuation amplitude, and reionization optical depth. The values of these six parameters are mostly not predicted by current theory; other possible parameters are fixed at "natural" values e.g. total density equals to 1.00 , neutrino masses are small enough to be negligible.

WMAP team, following the $\Lambda \mathrm{CDM}$ model, found the best $\Lambda \mathrm{CDM}$ fit parameters and based on them derived Cosmological parameters including Age of the Universe $A_{\tau}=13.772+0.059 \mathrm{Gyr}$ and Hubble parameter $H_{0}=69.32 \pm 0.8 \mathrm{~km} / \mathrm{s} \cdot \mathrm{Mpc} \quad[3]$.

\subsection{Newtonian Constant of Gravitation}

Table 1, borrowed from CODATA Recommended Values of the Fundamental Physical Constants, 2010, summarizes the results of measurements of the Newtonian constant of gravitation [4]: 
Table 1. Summary of the results of measurements of the Newtonian constant of gravitation $G$ relevant to the 2010 adjustment.

\begin{tabular}{|c|c|c|c|}
\hline Source & Method & $\begin{array}{c}\text { Value } \\
\left(10^{-11} \mathrm{~m}^{3} \cdot \mathrm{kg}^{-1} \cdot \mathrm{s}^{-2}\right)\end{array}$ & $\begin{array}{l}\text { Rel. stand. } \\
\text { uncert. ppm }\end{array}$ \\
\hline Luther and Towler (1982) & Fiber torsion balance, dynamic mode & $6.67248(43)$ & 64 \\
\hline $\begin{array}{c}\text { Karagioz and Izmailov } \\
\text { (1996) }\end{array}$ & Fiber torsion balance, dynamic mode & $6.6729(5)$ & 75 \\
\hline Bagley and Luther (1997) & Fiber torsion balance, dynamic mode & $6.67398(70)$ & 100 \\
\hline $\begin{array}{l}\text { Gundlach and Merkowitz } \\
\qquad(2000,2002)\end{array}$ & $\begin{array}{l}\text { Fiber torsion balance, } \\
\text { dynamic compensation }\end{array}$ & $6.674255(92)$ & 14 \\
\hline Quinn et al. (2001) & $\begin{array}{l}\text { Strip torsion balance, compensation } \\
\text { mode, static deflection }\end{array}$ & $6.67559(27)$ & 40 \\
\hline $\begin{array}{l}\text { Kleinevoss (2002); } \\
\text { Kleinevoss et al. (2002) }\end{array}$ & Suspended body, displacement & $6.67422(98)$ & 150 \\
\hline $\begin{array}{l}\text { Armstrong and Fitzgerald } \\
\qquad(2003)\end{array}$ & $\begin{array}{l}\text { Strip torsion balance, } \\
\text { compensation mode }\end{array}$ & $6.67387(27)$ & 40 \\
\hline Hu, Guo, and Luo (2005) & Fiber torsion balance, dynamic mode & $6.67228(87)$ & 130 \\
\hline Schlamminger et al. (2006) & Stationary body, weight change & $6.67425(12)$ & 19 \\
\hline $\begin{array}{l}\text { Luo et al. (2009); } \\
\text { Tu et al. (2010) }\end{array}$ & Fiber torsion balance, dynamic mode & $6.67349(18)$ & 27 \\
\hline Parks and Faller (2010) & Suspended body, displacement & $6.67234(14)$ & 21 \\
\hline
\end{tabular}

Observe that the values of $G$ vary significantly depending on Method. The disagreement in the values of $G$ obtained by the various teams far exceeds the standard uncertainties provided with the values.

Detailed analysis of these results shows that there are three groups of measurements. Inside each such group, the measurements are not mutually exclusive; however, measurements outside of a group contradict the entire group:

- The first such group consists of six measurements with the average value of

$$
G_{1}=6.67401 \times 10^{-11} \mathrm{~m}^{3} \cdot \mathrm{kg}^{-1} \cdot \mathrm{s}^{-2}
$$

and relative standard uncertainty $28.5 \mathrm{ppm}$ (ppm is one part per million);

- The second one consists of four measurements with the average value of

$$
G_{2}=6.67250 \times 10^{-11} \mathrm{~m}^{3} \cdot \mathrm{kg}^{-1} \cdot \mathrm{s}^{-2}
$$

and relative standard uncertainty $24 \mathrm{ppm}$;

- The third one consists of one measurement with the value of

$$
G_{3}=6.67539 \times 10^{-11} \mathrm{~m}^{3} \cdot \mathrm{kg}^{-1} \cdot \mathrm{s}^{-2}
$$

and relative standard uncertainty $40 \mathrm{ppm}$.

Clearly, the relative uncertainty of any such group is better than the uncertainty of the entire result set. $G_{1}, G_{2}, G_{3}$ has relative standard uncertainties that are about 4,5 , and 3 times smaller than the value of $120 \mathrm{ppm}$ for the average value of $G_{2010}$ :

$$
G_{2010}=6.67384 \times 10^{-11} \mathrm{~m}^{3} \cdot \mathrm{kg}^{-1} \cdot \mathrm{s}^{-2}
$$


The measurements falling into three groups are mutually exclusive; it is therefore likely that one group of measurements is correct, and the others are not.

\section{Ordinary Matter}

\subsection{Fundamental Parameter $Q$. Recommended Values of the Newtonian Parameter of Gravitation, Hubble's Parameter, Age of the World, and Temperature of the Microwave Background Radiation}

The constancy of the Universe Fundamental constants, including Newtonian constant of gravitation $G$, is now commonly accepted, although has never been firmly established as a fact. All conclusions on the constancy of $G$ are model-dependent [5]. In our opinion, it is impossible to either prove or disprove the constancy of $G$. Consequently, variability of $G$ with time can legitimately be explored. Alternative cosmological models describing the Universe with time-varying $G$ are widely discussed in literature (see e.g. [5] and references therein).

WUM is based on two parameters: dimensionless Rydberg constant $\alpha=\left(2 a R_{\infty}\right)^{1 / 3}$, where $R_{\infty}$ is Rydberg constant, $a$ is the basic unit of size (classical electron radius equals to: $\left.a_{0}=a / 2 \pi\right)$; and adimensionless time-varying parameter $Q$, which is a measure of a Size $R$ and Age $A_{t}$ of the World: $Q=R / a=A_{\tau} / t_{0}$, where $t_{0}=a / c$ is the basic unit of time and $c$ is the gravitodynamic constant.

In the present Epoch, the calculated value of $Q$ based on the average value of the Gravitational parameter in 2018 is:

$$
Q=0.759972 \times 10^{40}
$$

A commonly held opinion states that gravity has no established relation to other fundamental forces, so it does not appear possible to calculate it indirectly from other constants that can be measured more accurately, as is done in some other areas of physics. WUM holds that there indeed exist relations between all Q-dependent, time-varying parameters: Newtonian Parameter of Gravitation $G$, Hubble's parameter $H_{0}$, Age of the World $A_{\tau}$, Temperature of the microwave background radiation $T_{M B R}$, Critical energy density of the World $\rho_{c r}$, Photon minimum energy $E_{p h}$, etc. [5]. In frames of WUM, all Primary Cosmological parameters are inter-connected [7].

In accordance with WUM, the primary parameters of the World can be expressed as follows [5]:

- Newtonian parameter of gravitation $G$

$$
G=\frac{a^{2} c^{4}}{8 \pi h c} \times Q^{-1}
$$

- Hubble's parameter $H_{0}$

$$
H_{0}=\frac{c}{a} \times Q^{-1}
$$


- Age of the World $A_{c}$

$$
A_{\tau}=\frac{a}{c} \times Q
$$

- Temperature of the microwave background radiation $T_{M B R}$

$$
T_{M B R}=\frac{E_{0}}{k_{B}}\left(\frac{15 \alpha}{2 \pi^{3}} \frac{m_{e}}{m_{p}}\right)^{1 / 4} \times Q^{-1 / 4}
$$

where $k_{B}$ is Boltzmann constant; $m_{p}$ is the mass of a proton; $m_{e}$ is the mass of an electron and a basic unit of energy $E_{0}$ equals to $E_{0}=h c / a=0.070025267 \mathrm{GeV}$, where $h$ is Plank constant.

In 2013, the following two parameters were measured with the best precision: $G(120 \mathrm{ppm})$ and $T_{M B R}(210 \mathrm{ppm})$. At that time, we could calculate the value of $Q_{G}$ based on the average value of $G_{2010}$ :

$$
Q_{G}=0.760000 \times 10^{40}
$$

and using $Q_{G}$, substantially increase the precision of other parameters. With the help of WUM, more precise measurement of $T_{M B R}$ can help us narrow down the correct group of $G$ measurements. The right group can be selected once the relative standard uncertainty of the measurement of $T_{M B R}$ becomes significantly better than $30 \mathrm{ppm}$, but it is not the case. Then the choice of the correct group of $G$ measurements would appear to be a hopeless goal.

In frames of WUM, we succeeded to find the following equation for Fermi Coupling parameter $G_{F}[6]$ :

$$
\frac{G_{F}}{(\hbar c)^{3}}=\sqrt{30}\left(2 \alpha \frac{m_{e}}{m_{p}}\right)^{1 / 4} \frac{m_{p}}{m_{e}} \frac{1}{E_{0}^{2}} \times Q^{-1 / 4}
$$

where $\hbar$ is Dirac constant: $\hbar=h / 2 \pi$. We used the average value of $G_{F}$ with relative standard uncertainty $4.3 \mathrm{ppm}$ in 2010 and calculated the value of parameter $Q_{F}$

$$
Q_{F}=0.759960 \times 10^{40}
$$

Then the value of the predicted parameter $G$ in this case equals to

$$
G=6.67420 \times 10^{-11} \mathrm{~m}^{3} \cdot \mathrm{kg}^{-1} \cdot \mathrm{s}^{-2}
$$

that is close to the value of $G_{1}$ for the first group

$$
G_{1}=6.67401 \times 10^{-11} \mathrm{~m}^{3} \cdot \mathrm{kg}^{-1} \cdot \mathrm{s}^{-2}
$$

WUM calculates the value of the temperature of the microwave background radiation:

$$
T_{M B R}=2.72522 \mathrm{~K}
$$

that is in excellent agreement with experimentally measured value [2]:

$$
T_{\text {MBR }}=2.72548 \pm 0.00057 \mathrm{~K}
$$

We are not aware of any other model that allows calculation of MBR temperature with such accuracy. 
WUM calculates the value of the Hubble's parameter:

$$
H_{0}=68.7494 \mathrm{~km} / \mathrm{s} \cdot \mathrm{Mpc}
$$

which is in good agreement with the derived by WMAP team value [3]:

$$
H_{0}=69.32 \pm 0.8 \mathrm{~km} / \mathrm{s} \cdot \mathrm{Mpc}
$$

and with the newest value of the Hubble's parameter

$$
H_{0}=69.6 \pm 0.8( \pm 1.1 \% \text { stat }) \pm 1.7( \pm 2.4 \% \text { sys }) \mathrm{km} / \mathrm{s} \cdot \mathrm{Mpc}
$$

found by W. L. Freedman, et al., using the revised (and direct) measurement of the LMC (Large Magellanic Cloud) TRGB (Tip of the Red Giant Branch) extinction [8].

It is worth to note that the situation with the measurement accuracy of the Hubble's parameter in 2019 [9] looks the same as it was with the measurement accuracy of the gravitation parameter in 2013. We hope that WUM will prove helpful in determining the correct methods of measurement of the Hubble's parameter.

WUM calculates the Age of the World:

$$
A_{\tau}=14.2226 \mathrm{Gyr}
$$

that is much longer than the value derived by WMAP team

$$
A_{\tau}=13.772 \pm 0.059 \mathrm{Gyr}
$$

In frames of WUM, the difference between them 0.45 Gyr is the longevity of Dark Epoch [7].

To summarize: parameters $G, H_{0}, A_{\tau}$, and $T_{M B R}$ are all inter-connected. The first group of $G_{1}$ measurements is correct.

When these results were obtained, we sent the following letter to every member of the CODATA Task Group on Fundamental Physical Constants (TGFC) and every participant of the Royal Society Meeting [10]:

In 1937, Paul Dirac proposed a new basis for cosmology: the hypothesis of a variable gravitational "constant"; and later added the notion of continuous creation of matter in the World. My World-Universe Model follows these ideas, albeit introducing a different mechanism of matter creation. The proposed Model provides a mathematical framework based on a few basic assumptions, that allows to calculate the primary parameters of the World (its size, age, Hubble's parameter, the temperature of the cosmic microwave background radiation, masses of neutrinos and dark matter particles, etc.), in good agreement with the most recent measurements and observations. The Model published on viXra http://vixra.org/abs/1303.0077 v7.

Recently I published on viXra http://vixra.org/abs/1312.0179 v2 a new paper which gives the self-consistent set of $Q$-dependent, time varying values of the basic parameters of the World: Fermi Coupling parameter, Newtonian parameter of Gravitation, Hubble's parameter, Age of the World, and Temperature of the Microwave Background Radiation. It describes in detail the adjustment of the values of the parameters based on the World - Universe Model. The ob- 
tained set of values is recommended for consideration in CODATA Recommended Values of the Fundamental Physical Constants 2014.

Terry Quinn in the paper "Outcome of the Royal Society meeting on $G$ held at Chicheley Hall on 27 and 28 February 2014 to discuss 'The Newtonian constant of gravitation, a constant too difficult to measure?' concluded [11]:

"Thus, instead of simply calling for new determinations of $G$, it is suggested that an international advisory board be created, made up largely of those who have already carried out a $G$ experiment, to advise on the choice of method or methods, on the design of the experiment, on its construction and finally on the interpretation of the data and calculation of the results. This would be in contrast to the present situation in which outside criticism and comments can be brought to bear only when the experiment is finished and published when it is too late to affect the outcome. It is only by proceeding in this way that one might hope to obtain results that are demonstrably reliable".

At the time, CODATA stated the following value of $G_{2010}$ :

$$
G_{2010}=6.67384 \times 10^{-11} \mathrm{~m}^{3} \cdot \mathrm{kg}^{-1} \cdot \mathrm{s}^{-2}
$$

with relative uncertainty of $120 \mathrm{ppm}$. To the best of our knowledge, no breakthrough in $G$ measurement methodology has been achieved since. Nevertheless, in 2015 CODATA recommended a more precise value of the Newtonian constant of gravitation

$$
G_{2014}=6.67408 \times 10^{-11} \mathrm{~m}^{3} \cdot \mathrm{kg}^{-1} \cdot \mathrm{s}^{-2}
$$

with relative standard uncertainty 47 ppm [12]. In 2018 the recommendation improved further:

$$
G_{2018}=6.67430 \times 10^{-11} \mathrm{~m}^{3} \cdot \mathrm{kg}^{-1} \cdot \mathrm{s}^{-2}
$$

with relative standard uncertainty $22 \mathrm{ppm}$ [13]. Since 2013, the relative standard uncertainty of $G$ measurements reduced from $120 \mathrm{ppm}$ to $22 \mathrm{ppm}$ !

The variations of the average values of $G_{2014}$ and $G_{2018}$ around the predicted value of $G$ are:

$$
\frac{G_{2018}}{G_{2014}}=G_{-0.00012}^{+0.00010}
$$

Compare this result with the variations of the average values of $G_{2}$ and $G_{3}$ around the average value of $G$ in 2010 :

$$
\frac{G_{3}}{G_{2}}=G_{-0.00134}^{+0.00155}
$$

which are by order of magnitude larger than the variations of $G_{2014}$ and $G_{2018}$ around the predicted value of $G$.

It seems that CODATA considered the WUM recommendation that the first group of $G_{1}$ measurements is correct. In any case, the predicted by WUM in 2013 value of the Gravitational parameter is in an excellent agreement with its accepted value in 2014 [12] and in 2018 [13].

In 2014, WUM calculated the stationary temperature of Cosmic Large Grains 
based on the thermo-equilibrium, that corresponds to the FIRB temperature peak [14]:

$$
T_{\text {FIRB }}=\frac{E_{0}}{k_{B}}\left(\frac{15 \alpha}{4 \pi^{5}}\right)^{1 / 4} \times Q^{-1 / 4}
$$

and obtained $T_{\text {FIRB }}=28.955 \mathrm{~K}$, which is in good agreement with experimentally measured value of $29 \mathrm{~K}$ [1]. Comparing equations for $T_{M B R}$ and $T_{F I R B}$, we can find the relation between them [14]:

$$
T_{F I R B}=\left(3 \Omega_{e}\right)^{-1 / 4} \times T_{M B R}
$$

where $\Omega_{e}$ is the relative energy density of electrons in the Medium of the World in terms of the critical energy density $\rho_{c r}$ [5]:

$$
\Omega_{e}=\frac{2 \pi^{2} \alpha}{3} \frac{m_{e}}{m_{p}}
$$

\subsection{Missing Baryon Problem}

The Missing Baryon Problem related to the fact that the observed amount of baryonic matter did not match theoretical predictions. Observations by the Planck spacecraft in 2015, yielded a theoretical value for baryonic matter of $4.85 \%$ of the contents of the Universe [15]. However, directly adding up all the known baryonic matter produces a baryonic density slightly less than half of this [16]. The missing baryons are believed to be located in the warm-hot intergalactic medium.

The existence of the Medium of the World is a principal point of WUM. It follows from the observations of Intergalactic Plasma; Cosmic Microwave Background Radiation; Far-Infrared Background Radiation. There is no empty space (vacuum) in WUM. Inter-galactic voids discussed by astronomers are in fact examples of the Medium in its purest [5].

Detailed analysis of Intergalactic plasma carried out in 2013 [5] showed that the concentration of protons $n_{p}$ and electrons $n_{e}$ can be found by the following equation:

$$
n_{p}=n_{e}=\frac{2 \pi^{2}}{a^{3}} \frac{m_{e}}{m_{p}} \times Q^{-1}
$$

$\rho_{p}=n_{p} E_{p}$ is the energy density of protons in the Medium. The relative energy density of protons in the Medium $\Omega_{p}$ is then the ratio of $\rho_{p} / \rho_{c r}$ :

$$
\Omega_{p}=\frac{2 \pi^{2} \alpha}{3}=0.048014655
$$

According to WUM, the relative energy density of baryons in Macroobjects $\Omega_{M O}$ is:

$$
\Omega_{M O}=\frac{1}{2} \Omega_{p}=\frac{\pi^{2} \alpha}{3}=0.024007318
$$

The calculated values of $\Omega_{p}$ and $\Omega_{M O}$ are in good agreement with their 
2015 estimations [15] [16].

In our opinion, direct measurements of the Intergalactic plasma parameters can be done by investigations of Fast Radio Bursts, which are millisecond duration radio signals originating from distant galaxies. These signals are dispersed according to a precise physical law and this dispersion is a key observable quantity which, in tandem with a redshift measurement, can be used for fundamental physical investigations [17].

The dispersion measure and redshift, carried out in 2016 by E. F. Keane, et al., provide a direct measurement of the cosmic density of ionized baryons in the intergalactic medium $\Omega_{I G M}$ [17]:

$$
\Omega_{\text {IGM }}=4.9 \pm 1.3 \%
$$

that is in excellent agreement with the predicted by WUM value of $\Omega_{p}$.

Using the equation for $n_{e}$, we calculated the value of photons' time delay [18]:

$$
\Delta t_{p h}^{c a l}=2.189 \times\left(\frac{v}{1 \mathrm{GHz}}\right)^{-2}
$$

which is in good agreement with experimentally measured value [17]:

$$
\Delta t_{p h}^{e x p}=2.438 \times\left(\frac{v}{1 \mathrm{GHz}}\right)^{-2}
$$

To summarize: the values of the Intergalactic plasma parameters predicted by WUM in 2013 are confirmed by experiments conducted in 2016.

\subsection{Energy-Varying Photons}

Analysis of Intergalactic plasma shows that the value of the lowest plasma frequency $v_{p l}$ is [5]:

$$
v_{p l}=\frac{c}{a}\left(\frac{m_{e}}{m_{p}}\right)^{1 / 2} \times Q^{-1 / 2}=4.5322 \mathrm{~Hz}
$$

Photons with energy smaller than $E_{p h}=h v_{p l}$ cannot propagate in plasma, thus $h v_{p l}$ is the smallest amount of energy a photon may possess. Following L. Bonetti, et al. [19] we can call this amount of energy the rest energy of photons that equals to

$$
E_{p h}=\left(\frac{m_{e}}{m_{p}}\right)^{1 / 2} E_{0} \times Q^{-1 / 2}=1.8743 \times 10^{-14} \mathrm{eV}
$$

The above value, predicted by WUM in 2013, is in good agreement with the value

$$
E_{p h} \lesssim 2.2 \times 10^{-14} \mathrm{eV}
$$

obtained by L. Bonetti, et al. in 2017 [19]. It is more relevant to call $E_{p h}$ the minimum energy of photons which can pass through the Intergalactic plasma. It is worth to note that $E_{p h}$ is varying in time: $E_{p h} \propto \tau^{-1 / 2}$. 


\subsection{Mass-Varying Neutrinos}

It is now established that there are three different types of neutrino: electronic $v_{e}$, muonic $v_{\mu}$, and tauonic $v_{\tau}$. Neutrino oscillations imply that neutrinos have non-zero masses. The neutrino was postulated first by Wolfgang Pauli in 1930 to explain how beta decay could conserve energy, momentum, and angular momentum (spin). But we still don't know the values of neutrino masses. Experimentalists are measuring $\Delta m_{\text {sol }}^{2}$ and $\Delta m_{\text {atm }}^{2}$ which are mass splitting for solar and atmospheric neutrinos respectively.

The situation with Dark Matter Particles (DMPs) is similar: we can investigate them indirectly by the analysis of gamma-rays and X-rays irradiated as the result of DMPs self-annihilation (see Section 4.1).

In 2013, WUM predicted the following values of neutrino mass eigenstates:

$$
\begin{gathered}
m_{v_{\mu}}=m_{0} \times Q^{-1 / 4} \cong 7.5 \times 10^{-3} \mathrm{eV} / \mathrm{c}^{2} \\
m_{v_{\tau}}=6 m_{v_{\mu}} \cong 4.5 \times 10^{-2} \mathrm{eV} / \mathrm{c}^{2} \\
m_{v_{e}}=\frac{1}{24} m_{v_{\mu}} \cong 3.1 \times 10^{-4} \mathrm{eV} / \mathrm{c}^{2}
\end{gathered}
$$

where $m_{0}$ is a basic unit of mass: $m_{0}=h / a c$. The sum of the predicted neutrino masses

$$
\Sigma m_{v} \cong 0.053 \mathrm{eV} / c^{2}
$$

is in good agreement with the value of $0.06 \mathrm{eV} / c^{2}$ discussed in literature [20]. It is worth to note that $m_{v}$ is varying in time: $m_{v} \propto \tau^{-1 / 4}$.

As the conclusion, in 2013-2014 WUM gave the following results for Ordinary Matter:

- Calculated the values of parameters $H_{0}, T_{M B R}, T_{F I R B}$, that are in good agreement with experimental results;

- Predicted the values of cosmological parameters $G, n_{p}, \Omega_{p}, E_{p h}$, which were confirmed experimentally in 2015-2018;

- Predicted the values of neutrino masses.

\section{Dark Matter}

\subsection{Multicomponent Dark Matter}

Dark Matter (DM) is among the most important open problems in both cosmology and particle physics. Dark Matter Particles (DMPs) might be observed in Centers of Macroobjects has drawn many new researchers to the field in the last forty years [7]. Important cosmological problems like Dark Matter and Dark Energy could be, in principle, solved through extended gravity. This is stressed, for example, in the famous paper of Prof. C. Corda [21].

Two-component DM system consisting of bosonic and fermionic components is proposed for the explanation of emission lines from the bulge of Milky Way galaxy. C. Boehm, P. Fayet, and J. Silk analyze the possibility of two coannihilating neutral and stable DMPs: a heavy fermion for example, like the lightest neu- 
tralino $(>100 \mathrm{GeV})$ and the other one a possibly light spin-0 particle $(\sim 100 \mathrm{MeV})$ [22].

WUM proposes multicomponent DM system consisting of two couples of coannihilating DMPs: a heavy DM fermion-DMF1 $(1.3 \mathrm{TeV})$ and a light spin-0 boson-DIRAC $(70 \mathrm{MeV})$ that is a dipole of Dirac's monopoles; a heavy fermion-DMF2 $(9.6 \mathrm{GeV})$ and a light spin-0 boson-ELOP $(340 \mathrm{keV})$ that is a dipole of preons with electrical charge e/3; a self-annihilating fermion-DMF3 (3.7 keV) and a fermion DMF4 $(0.2 \mathrm{eV})$.

WUM postulates that masses of DMFs and bosons are proportional to $m_{0}$ multiplied by different exponents of $\alpha$ and can be expressed with the following formulae [5] [7]:

DMF1 (fermion): $m_{D M F 1}=\alpha^{-2} m_{0}=1.3149950 \mathrm{TeV}$

DMF2 (fermion): $m_{D M F 2}=\alpha^{-1} m_{0}=9.5959823 \mathrm{GeV}$

DIRAC (boson): $m_{\text {DIRAC }}=\alpha^{0} m_{0}=70.025267 \mathrm{MeV}$

ELOP (boson): $m_{E L O P}=2 / 3 \alpha^{1} m_{0}=340.6660 \mathrm{keV}$

DMF3 (fermion): $m_{D M F 3}=\alpha^{2} m_{0}=3.7289402 \mathrm{keV}$

DMF4 (fermion): $m_{D M F 4}=\alpha^{4} m_{0}=0.19857111 \mathrm{eV}$

The values of mass of DMF1, DMF2, DMF3 fall into the ranges estimated in literature for neutralinos, WIMPs, and sterile neutrinos respectively. DMF1, DMF2 and DMF3 partake in the self-annihilation interaction with strength equals to $\alpha^{-2}, \alpha^{-1}$ and $\alpha^{2}$ respectively [5].

The widely discussed models for nonbaryonic DM are based on the Cold DM hypothesis, and corresponding particles are commonly assumed to be WIMPs, which interact via gravity and any other force (or forces), potentially not part of the standard model itself, which is as weak as or weaker than the weak nuclear force, but also non-vanishing in its strength. It follows that a new weak force needs to exist, providing interaction between DMPs. The strength of this force exceeds that of gravity, and its range is considerably greater than that of the weak nuclear force [7].

In WUM, strength of the proposed weak interaction is characterized by the parameter $G_{W}$ :

$$
G_{W}=G_{0} \times Q^{-1 / 4}
$$

where $G_{0}=a^{2} c^{4} / 8 \pi h c$ is an extrapolated value of $G$ at the Beginning of the World $(Q=1)$. In the present epoch, $Q=0.759972 \times 10^{40}$, and thus $G_{W}$ is about 30 orders of magnitude greater than $G$. The range of the weak interaction $R_{W}$ in the present Epoch equals to:

$$
R_{W}=a \times Q^{1 / 4}=1.65314 \times 10^{-4} \mathrm{~m}
$$

that is much greater than the range of the weak nuclear force. The predicted Weak Interaction between DMPs provides integrity of all DM shells in all Macroobjects. In our view, the foretold weak interaction between particles DMF3 provides integrity of Fermi Bubbles [7].

The signatures of DMPs annihilation with predicted masses of $1.3 \mathrm{TeV}, 9.6$ 
$\mathrm{GeV}, 70 \mathrm{MeV}, 340 \mathrm{keV}$, and $3.7 \mathrm{keV}$, which are calculated independently of astrophysical uncertainties, are found in spectra of the diffuse gamma-ray background and the emission of various Macroobjects in the World. The correlation between different emission lines in spectra of Macroobjects is connected to their structure, which depends on the composition of the Core and surrounding shells made up of DMPs. Thus, the diversity of Very High Energy gamma-ray sources in the World has a clear explanation [23].

\subsection{Predicted Distribution of the World's Energy Density}

According to WUM, the total DMF4 relative energy density $\rho_{D M F 4}$, in terms of proton energy density in the Medium of the World $\rho_{p}=2 \pi^{2} \alpha \rho_{c r} / 3$, equals to [7]:

$$
\rho_{D M F 4}=\frac{45}{\pi} \rho_{p}=30 \pi \alpha \rho_{c r}=0.68775927 \rho_{c r}
$$

Our Model holds that the energy density of all types of self-annihilating DMPs is proportional to $\rho_{p}$. In all, there are 5 different types of self-annihilating DMPs: DMF1, DMF2, DIRAC, ELOP, and DMF3. Then the total energy density of DM $\rho_{D M}$ is

$$
\rho_{D M}=5 \rho_{p}=0.24007327 \rho_{c r}
$$

The total baryonic energy density $\rho_{B}$ is:

$$
\rho_{B}=1.5 \rho_{p}
$$

The sum of electron and MBR energy densities $\rho_{\text {eMBR }}$ equals to:

$$
\rho_{\text {eMBR }}=1.5 \frac{m_{e}}{m_{p}} \rho_{p}+2 \frac{m_{e}}{m_{p}} \rho_{p}=3.5 \frac{m_{e}}{m_{p}} \rho_{p}
$$

We take energy density of neutrinos $\rho_{v}$ to equal:

$$
\rho_{v}=\rho_{\text {MBR }}
$$

For FIRB radiation energy density $\rho_{\text {FIRB }}$ we take

$$
\rho_{\text {FIRB }}=\frac{1}{5 \pi} \frac{m_{e}}{m_{p}} \rho_{p} \approx 0.032 \rho_{\text {MBR }}
$$

which corresponds to the value of $0.034 \rho_{M B R}$ calculated by E. L. Wright [24]. Then the energy density of the World $\rho_{W}$ in Luminous Epoch equals to the theoretical critical energy density $\rho_{c r}$

$$
\rho_{W}=\left[\frac{45}{\pi}+6.5+\left(5.5+\frac{1}{5 \pi}\right) \frac{m_{e}}{m_{p}}\right] \rho_{p}=\rho_{c r}
$$

From this equation we can calculate the value of $1 / \alpha$ using electron-to-proton mass ratio $m_{e} / m_{p}$

$$
\frac{1}{\alpha}=\frac{\pi}{15}\left[450+65 \pi+(55 \pi+2) \frac{m_{e}}{m_{p}}\right]=137.03600
$$

which is in excellent agreement with the commonly adopted value of 137.035999. 
It follows that there is a direct correlation between constants $\alpha$ and $m_{e} / m_{p}$ expressed by the obtained equation. As shown, $m_{e} / m_{p}$ is not an independent constant but is instead derived from $\alpha$ [7].

As the conclusion, according to WUM:

- The World's energy density is inversely proportional to the dimensionless time-varying parameter $Q \propto \tau$ in all cosmological times;

- The particles relative energy densities are proportional to constant $\alpha$ in Luminous Epoch.

\section{Hypersphere World-Universe Model}

The sciences do not try to explain, they hardly even try to interpret, they mainly make models. By a model is meant a mathematical construct, which, with addition of certain verbal interpretations describes observed phenomena. The justification of such a mathematical construct is solely and precisely that it is expected to work.

John von Newmann

The Hypersphere WUM is the only cosmological model in existence that [7]:

- Is consistent with the Law of conservation of angular momentum, and answers the following questions: why is the orbital momentum of Jupiter larger than rotational momentum of Sun, and how did Milky Way galaxy and Solar system obtain their substantial orbital angular momentum?

- Reveals the Inter-connectivity of primary cosmological parameters of the World (Age, Size, Hubble's parameter, Newtonian parameter of gravitation, Critical energy density, Concentration of Intergalactic Plasma, Temperature of the Microwave Background Radiation, Temperature of the Far-Infrared Background Radiation Peak) and calculates their values, which are in good agreement with experimental results;

- Considers Fermi Bubbles (FBs) that built up from Dark Matter Particles (DMPs), and explains X-rays and gamma-rays radiated by FBs as a result of DMPs annihilation;

- Solves Coronal heating problem that relates to the question of why the temperature of the Solar corona is millions of degrees higher than that of the photosphere. In WUM, the Solar corona is made up of DMPs, and the plas$\mathrm{ma}$ is the result of their annihilation. The Solar corona resembles a honeycomb filled with plasma. The Geocorona and Planetary Coronas possess features similar to these of the Solar Corona;

- Explains the diversity of Very High Energy gamma-ray sources in the World in frames of the proposed Macroobject (MO) Shell Model, which describes Cores of MOs as Nuclei made up of annihilating Dark Matter Fermions (DMFs) surrounded by shells containing other DMPs;

- Explains the diversity of gravitationally-rounded objects (planets and moons in Solar system) and their internal heat through annihilation of DMFs in their Cores. 
WUM envisions the following picture of creation and evolution of the World [7]:

- Overspinning (surface speed at equator exceeding escape velocity) DM Cores of Superclusters initiate creation of all World's Macrostructures;

- The outer shells of Supercluster's Cores are composed of DMF4 with mass of $0.2 \mathrm{eV}$ and total energy density of $68.8 \%$ of the overall energy density of the World;

- Proposed Weak Interaction between DMPs provides the integrity of Dark Matter (DM) Cores of all MOs;

- DMF4 outer shells of Supercluster's Cores are growing to the critical mass during Dark Epoch lasting from the Beginning of the World (14.22 billion years ago) for 0.45 billion years;

- Luminous Galaxies and Extrasolar Systems arise due to Rotational Fission of Overspinning Supercluster's Cores and annihilation of DMPs;

- Macrostructures of the World form from Superclusters down to Galaxies, Extrasolar systems, planets, and moons. Formation of galaxies and stars is not a process that concluded ages ago; instead, it is ongoing in the Luminous Epoch;

- Luminous Epoch spans from 0.45 billion years up to the present Epoch for 13.77 billion years. The Big Bang discussed in the standard cosmological model is, in our view, the transition from Dark Epoch to Luminous Epoch.

In frames of WUM, Time and Space are closely connected with Mediums' impedance and gravitomagnetic parameter. It follows that neither Time nor Space could be discussed in absence of the Medium. The gravitational parameter $G$ that is proportional to the Mediums' energy density can be introduced only for the Medium filled with Matter. Gravity, Space and Time are all emergent phenomena [7].

WUM confirms the Supremacy of Matter postulated by Albert Einstein:

"When forced to summarize the theory of relativity in one sentence: time and space and gravitation have no separate existence from matter".

WUM is based on two parameters only: dimensionless Rydberg constant $\alpha$ and dimensionless time-varying quantity $Q$. In WUM we often use well-known physical parameters, keeping in mind that all of them can be expressed through the Basic Units of time $t_{0}$, size $a$, and energy $E_{0}$. For example, $c=a / t_{0}$ and $h=E_{0} \times t_{0}$. Taking the relative values of physical parameters in terms of the Basic Units we can express all dimensionless parameters of the World through two parameters $\alpha$ and $Q$ in various rational exponents, as well as small integer numbers and $\pi$ [25].

There are no Fundamental Physical constants in WUM. In our opinion, constant $\alpha$ and quantity $Q$ should be named "Universe Constant" and "World Parameter" respectively.

The Hypersphere World-Universe Model successfully describes Primary Cosmological parameters and their relationships, ranging in scale from cosmo- 
logical structures to elementary particles. WUM predicted in 2013 the values of cosmological parameters $G, n_{p}, \Omega_{p}, E_{p h}$, which were confirmed experimentally in 2015-2018. The Model allows for precise calculation of values that were only measured experimentally earlier and makes verifiable predictions.

\section{Acknowledgements}

I'm grateful to my son Ilya Netchitailo who has reviewed and edited this work.

\section{Conflicts of Interest}

The author declares no conflicts of interest regarding the publication of this paper.

\section{References}

[1] Lagache, G., et al. (1999) First Detection of the WIM Dust Emission. Implication for the Cosmic Far-Infrared Background. arXiv:Astro-ph/9901059.

[2] Fixsen, D.J. (2009) The Temperature of the Cosmic Microwave Background. arXiv:0911.1955. https://doi.org/10.1088/0004-637X/707/2/916

[3] Bennett, C.L., et al. (2013) Nine-Year Wilkinson Microwave Anisotropy Probe (WMAP) Observations: Final Maps and Results. arXiv:1212.5225v3.

[4] Mohr, P.J., Taylor, B.N. and Newell, D.B. (2012) CODATA Recommended Values of the Fundamental Physical Constants: 2010. arXiv:1203.5425. https://doi.org/10.1063/1.4724320

[5] Netchitailo, V.S. (2013) Word-Universe Model. viXra:1303.0077.

[6] Netchitailo, V.S. (2013) Fundamental Parameter Q. Recommended Values of the Newtonian Parameter of Gravitation, Hubble's Parameter, Age of the World, and Temperature of the Microwave Background Radiation. viXra:1312.0179.

[7] Netchitailo, V. (2019) Dark Matter Cosmology and Astrophysics. Journal of High Energy Physics, Gravitation and Cosmology, 5, 999-1050. https://doi.org/10.4236/jhepgc.2019.54056

[8] Freedman, W.L., et al. (2020) Calibration of the Tip of the Red Giant Branch (TRGB). arXiv:2002.01550. https://doi.org/10.3847/1538-4357/ab7339

[9] Conover, E. (2019) Debate over the Universe's Expansion Rate May Unravel Physics. Is It a Crisis? Science News.

https://www.sciencenews.org/article/debate-universe-expansion-rate-hubble-consta nt-physics-crisis

[10] Quinn, T., Speake, C. and Luo, J. (2014) The Newtonian Constant of Gravitation, a Constant too Difficult to Measure? The Royal Society Meeting, London. https://royalsociety.org/science-events-and-lectures/2014/gravitation/

[11] Quinn, T. (2014) Outcome of the Royal Society Meeting on G Held at Chicheley Hall on 27 and 28 February 2014 to Discuss 'The Newtonian Constant of Gravitation, a Constant too Difficult to Measure? Philosophical Transactions of the Royal Society A, 372, No. 2026. https://doi.org/10.1098/rsta.2014.0286

[12] Mohr, P.J., Newell, D.B. and Taylor, B.N. (2015) CODATA Recommended Values of the Fundamental Physical Constants: 2014. arXiv:1507.07956. https://doi.org/10.6028/NIST.SP.961r2015

[13] NIST (2018) Codata Recommended Values of The Fundamental Physical Con- 
stants: 2018. https://physics.nist.gov/cuu/pdf/wall 2018.pdf

[14] Netchitailo, V.S. (2014) World-Universe Model. Cosmic Far-Infrared Background. viXra:1412.0265.

[15] Ade, P.A.R., et al. (2015) Planck 2015 Results. XIII. Cosmological Parameters. arXiv:1502.01589.

[16] Ferguson, H.C. The Case of the "Missing Baryons". https://archive.stsci.edu/hut/astro2/astro2 science/starburst.html

[17] Keane, E.F., et al. (2016) A Fast Radio Burst Host Galaxy. arXiv:1602.07477. https://doi.org/10.1038/nature17140

[18] Netchitailo, V. (2017) Burst Astrophysics. Journal of High Energy Physics, Gravitation and Cosmology, 3, 157-166. https://doi.org/10.4236/jhepgc.2017.32016

[19] Bonetti, L., et al. (2017) FRB 121102 Casts New Light on the Photon Mass. arXiv:1701.03097. https://doi.org/10.1016/j.physletb.2017.03.014

[20] Battye, R.A. and Moss, A. (2013) Evidence for Massive Neutrinos from CMB and Lensing Observations. arXiv: 1308.5870.

[21] Corda, C. (2009) Interferometric Detection of Gravitational Waves: The Definitive Test for General Relativity. International Journal of Modern Physics, 18, 2275-2282. https://doi.org/10.1142/S0218271809015904

[22] Boehm, C., Fayet, P. and Silk, J. (2003) Light and Heavy Dark Matter Particles. arXiv:0311143. https://doi.org/10.1103/PhysRevD.69.101302

[23] Netchitailo, V.S. (2014) World-Universe Model. Multicomponent Dark Matter. Cosmic Gamma-Ray Background. viXra:1406.0018.

[24] Wright, E.L. (2001) Cosmic InfraRed Background Radiation. http://www.astro.ucla.edu/ wright/CIBR/

[25] Netchitailo, V. (2020) World-Universe Model: Alternative to Big Bang Model. Journal of High Energy Physics, Gravitation and Cosmology, 6, 133-158. https://doi.org/10.4236/jhepgc.2020.61012 